\title{
Examination of the Interface of \\ a Model Adhesive Joint by Surface Analysis: \\ A Study by XPS and ToF-SIMS
}

\author{
Marie-Laure Abel* and John F Watts \\ The Surface Analysis Laboratory \\ Surrey Materials Institute and Faculty of Engineering \& Physical Sciences \\ University of Surrey \\ Guildford Surrey GU2 7XH UK
}

\begin{abstract}
:
Model samples of the interface of an adhesive joint containing small levels of aminopropyl triethoxysilane (APS) have been prepared in order to examine the interface formed with an aluminium substrate. X-ray photoelectron (XPS) and time of flight secondary ion mass spectrometry (ToF-SIMS) has been used to analyse and image the interface region in between the aluminium and an epoxy adhesive in order to ascertain the reactions by the organosilane, present as a minor component within the system.

It was found that APS was present at the interface between the adhesive and the substrate and that it had reacted with the substrate forming a covalent bond and was also crosslinked within the adhesive. Evidence of near to full hydrolysis of APS is also present within the spectra.
\end{abstract}

Keywords: Organosilanes, epoxy adhesive, interface, XPS, ToF-SIMS

Corresponding author: m.abel@surrey.ac.uk 


\section{Introduction}

Adhesive bonding technology is of foremost importance for the aerospace industry. Light-weight alloys, usually based on aluminium, are used for this type of application and usually a pretreatment has to be used to prepare the surface in order to create the appropriate roughness and chemistry to allow adhesive bonding to be successful. However, some of the treatments still involve the use of chromium rinses based on $\mathrm{Cr}(\mathrm{VI})$ which is highly toxic and also necessitates specific and expensive recycling of the chemicals and waste solutions employed for the process. One alternative consists of using organosilane based adhesion promoters such as $\gamma$-aminopropyl triethoxysilane (APS). It is known that the application of silanes on a given substrate can improve joint durability and also that silanes can form covalent bonds with hydroxyl functionalities present on the metal surface.

The obvious next step is to incorporate such an organosilane in a formulation rather than using it as a primer as this will reduce process steps in the joint fabrication and also simplifies adhesive application. Epoxy formulations of this type are already available as commercial products and it has been established that the incorporation of silanes improves the strength and durability of bonded joints. Until recently, it has been assumed that the mode of action is similar to that of an adhesion promoter when used as a primer and that the silane diffuses towards the interface where it undergoes a reaction with the hydrated metal surface to form a covalent bond. In reality, and although this assumption is logical, the exact mode of action is not well known and the concentration of the silane is chosen on an ad-hoc basis.

In order to understand the reactions that a silane undergoes as well as its diffusion behaviour, when incorporated in an adhesive formulation, model samples have been prepared with aluminium foil encased in a typical epoxy formulation adhesive containing a $0.5 \%(\mathrm{w} / \mathrm{w})$ concentration of APS. The interface between aluminium and adhesive has been examined with both XPS and ToF-SIMS.

\section{Experimental}

\section{1. Preparation of model adhesive samples}

A formulation of epoxy adhesive was prepared based on a two-part toughened epoxy adhesive formulation containing $0.5 \%(\mathrm{w} / \mathrm{w})$ of aminopropyltrimethoxy silane (APS) 
(Figure 1). The adhesive formulation was based on an Araldite 420 formulation which was modified from its usual silane concentration. No further details are available because the formulation is proprietary. To allow for unambiguous assignment of any silicon related signal to the organosilane, the formulation was prepared without the usual silica thixotrope or the glass beads used to define the glue line thickness. To represent an adhesive joint between aluminium and this adhesive, a model sample, referred to as "sandwich sample", was prepared by encasing an aluminium foil between two layers of adhesive. The foil used was a typical household foil which was not pretreated before encasing within the uncured adhesive. The formulation was prepared in typical industrial conditions and although the authors acknowledge that the amount of moisture within the components important, it is not known for this particular system. The sample was subsequently cured overnight at room temperature followed by two hours at $60^{\circ} \mathrm{C}$. A histological microtome was used to cut the sample mounted with a taper using the ultra-low angle microtomy method as described by Hinder et al $[1,2]$. In this work an angle of 0.33 degrees was used. Figure 2 provides a schematic of the so-called "sandwich" sample (2a) together with areas of analysis by ToF-SIMS as well as a side elevation (2b) indicating the depth $\mathrm{d}$ corresponding to the distance on the XPS concentration profiles.

\section{2. Surface analysis}

XPS analysis was performed in the form of a linescan analysis using a Thermo Scientific Sigma Probe spectrometer using a spot size of 100 micrometres, of the monochromated aluminium X-ray source with a step-size of 125 microns. A pass energy of $150 \mathrm{eV}$ was used to collect the optimum signal intensity and the following spectral regions were collected: $\mathrm{C} 1 \mathrm{~s}, \mathrm{O} 1 \mathrm{~s}, \mathrm{Si} 2 \mathrm{p}, \mathrm{N} 1 \mathrm{~s}, \mathrm{~A} 12 \mathrm{p}$ and Na1s. Concentration

profiles were subsequently constructed using the quantification obtained for particular elements and are presented from the aluminium/adhesive interface towards the bulk of the adhesive material. Those elements were aluminium, carbon and silicon. This choice was made because of the clarity of the data and to avoid the repetition induced by similar profiles such as carbon and nitrogen. All data were obtained with the manufacturer's software Avantage v2.18.

ToF-SIMS analyses were performed on the interface region of the microtomed sample using a TOF.SIMS 5 instrument from ION-TOF GmbH (Münster, Germany). Images 
were recorded using the high current bunched mode at $8 \mathrm{keV}$ with a $\mathrm{Bi}_{3}{ }^{+}$primary ion beam over an area of $500 \times 500 \mu \mathrm{m}^{2}$. This raster size is equivalent to a difference in depth of approximately $3 \mu \mathrm{m}$ in the environs of the interface in the direction in which the taper has been cut. Using the same mode, high mass resolution spectra were also recorded from two discrete areas of $100 \times 100 \mu \mathrm{m}^{2}$. Three ToF-SIMS analysis areas are identified in Figure 2(b), Areas 1, 2 and 3. Area 1 is where the ToF-SIMS images have been recorded from and within this zone three regions of interest (ROI) were defined; ROI 1, ROI 2 and ROI 3. A region of interest provides a means of reconstructing a spectrum from a user selected region of an image (in this case a $500 \mathrm{x}$ $500 \mu \mathrm{m}^{2}$ area of the sample). It may be chosen as a function of the intensity of a particular ion (such as $\mathrm{Al}^{+}$) or by tracing lines within the map region to define a zone of interest deduced from either a total ion or a mass selected image. Areas 2 and 3 of Figure 2(b) were large area $\left(100 \times 100 \mu \mathrm{m}^{2}\right)$ ToF-SIMS spectra at a region $890 \mu \mathrm{m}$ away from the interface within the polymer (Area 2) or at the interface (Area 3) itself.

\section{Results}

\section{1. XPS analysis}

Figure 3 shows concentration profiles for the elements: aluminium, carbon, silicon as well as a curve of the concentration ratio of silicon to nitrogen. The concentrations of the elements were plotted as a depth profile against the recalculated distance depth of the analysis obtained through microtoming. This value is the actual linescan distance multiplied by the sine of the angle of cut. In this work XPS is primarily used to provide concentrations profiles as the high pass energy employed in this work does not allow for high resolution spectra. The profile of silicon is of particular interest as it illustrates the diffusion of the silane within the system. Other elements are of major interest as for example aluminium which corresponds to the encased foil and carbon that will be in high amount when analysing a region of pure adhesive.

The first profile to examine is that of aluminium (Figure 3(a)); as anticipated a high concentration of aluminium is found at the beginning of the profile indicating that the experiment started indeed from pure aluminium. This concentration then sharply decreases and finally falls to zero once analysis position is within a region where the aluminium is completely covered by the adhesive. The carbon profile (Figure 3(b)) 
starts from a level of 27 at.\% which is consistent with a very clean aluminium surface and also with the microtomy having seemingly exposed the pure aluminium within the sample. The profile then evolves in an increase into the amount of carbon expected for the pure adhesive material at around 79at.\%. The nitrogen profile (not shown here) follows approximately the same variation albeit with a less intense signal which again is consistent with the analysis going through the interface with the metal as nitrogen in small levels originates from APS and at higher concentrations from the crosslinker incorporated within the formulation as well as the toughening agent. The silicon signal (Figure 3(c)) is a marker for the adhesion promoter APS and is more specific than nitrogen which is also contained in the adhesive. Although APS was included within the formulation at a very low concentration, it is possible to find it at the interface between the metal and the adhesive and therefore show that the molecule has indeed migrated to where it is expected to achieve the most benefit. The front of diffusion as indicated by the silicon profile is approximately 5 micrometres away from the substrate. The size of the interphase, between substrate and adhesive is provided by the difference between the values at $16 \%$ and $84 \%$ of the plateau value and is measured at 1.5 micrometres. Figure 3(d) shows the ratio of the silicon to the nitrogen intensity. Whenever this ratio is equal to one this may be considered as an indication that the APS molecule is present without mixing with the adhesive. It is rather striking that the region of the curve where this ratio is equal to one is close to the size of the interphase itself and is indicated by data points included within the shaded rectangle in Figure 3(d).

\section{2. ToF-SIMS analysis}

Figure 4 shows a comparison of positive ToF-SIMS spectra recorded at the interface region (Area 3) and approximately 890um away (Area 2) together with assignment of the main ions. Only these two spectra are presented as the spectrum obtained from Area 1 presented in this work is very similar to the spectrum of Area 3, one of the main differences being the amount of aluminium. It is fairly obvious that the lower spectrum of Figure 4, Area 2, is dominated by PDMS ions; at this stage it is unclear whether the origin of those ions is due to contamination or whether they are the result of polymerisation of APS. Other ions specific of the epoxy based adhesive are also present such as $\mathrm{m} / \mathrm{z}=135 \mathrm{u}\left(\mathrm{C}_{9} \mathrm{H}_{11} \mathrm{O}^{+}\right)$or $\mathrm{m} / \mathrm{z}=57 \mathrm{u}\left(\mathrm{C}_{3} \mathrm{H}_{5} \mathrm{O}^{+}\right)$. The upper spectrum does not exhibit any PDMS ions but mostly $\mathrm{C}_{\mathrm{x}} \mathrm{H}_{\mathrm{y}}$ ions which may be assigned to the 
rubber phase added to the system to provide toughness. Those ions are presumably intense compared to epoxy based ions because they originate from a part of the adhesive which can still be considered like a thermoplastic and hence will provide ions more easily than this thermoset phase. Additionally one may observe silane related ions such as $\mathrm{SiOH}^{+}$at $\mathrm{m} / \mathrm{z}=45 \mathrm{u}$. Figure 5(a) provides the total ion signal image of the interface region in a similar area to top spectrum. The interface is oriented at an angle of approximately 45 degrees and from bottom left to top right. The image reveals a smooth central area with the adhesive present on either side. However only the top left part of the image will be considered as it is the region where the adhesive is thin and just covers the foil. The other side of the adhesive (bottom right) unfortunately provides an erroneous signal. It is a thick piece of polymeric material and as it charges significantly, would require a very different setting of the reflector voltage to obtain credible data. Indeed, this region appears as black in Figure 5(a) even though it is at the same height as the rest of the sample and clearly not a void. All subsequent images are presented normalised to the total ion signal to avoid any significant effect as a result due to roughness or morphology of the sample and, as justified above, the bottom right region of thick adhesive will not be considered at all in reconstruction of spectra or discussion of the data. Figure 5(b) shows the image obtained for $\mathrm{Al}^{+}$and it shows that the foil is covered by a layer of material which progressively disappears as we translate from the adhesive region to a strong signal of aluminium following the heat scale from orange to yellow. This is indicated in the Figure by an arrow progressing from the low to high intensity region of aluminium. As PDMS or PDMS like peaks are present and are a major component of the spectrum obtained by integrating the image area, an image was created to localise where PDMS is mostly present on the sample and this is illustrated in Figure 5(c) where the signal of aluminium has been superimposed with the image result of the addition of the most intense PDMS signals as well as an intermediate region for which the signal of PDMS is smaller and the amount of other organic material is higher. This Figure shows that PDMS is mostly present in the region where the adhesive covers sufficiently the aluminium for it not to yield any signal. This seems to imply that any examination of the interaction of APS on aluminium remains valid in the region of the interface where aluminium is visible using ToF-SIMS. 
To successfully bond with the aluminium foil, the silane present within the system should not only migrate to the interface but also undergo several specific reactions; the most important two are hydrolysis where the silane loses its alkoxy functionalities which are in turn transformed into silanols and bonding with the substrate in a condensation reaction. In parallel to these, another condensation reaction is possible when the silane reacts with itself and it may be described as a type of crosslinking. Those are illustrated in Figure 6(a) to (c) respectively and at this stage it might be useful to recall that all images have been recorded at low spatial resolution but high mass resolution, hence the ions assignments, whether for spectra or images, are provided for an exact mass. Figure 6(a) shows the hydrolysis reaction of APS and circled is also shown which part of the hydrolysed molecule can perfectly illustrate the presence or absence of full hydrolysis. The ion corresponding to this part of the molecule may occur if the bond between silicon and the carbon chain is broken which is fairly likely as the silicon is the centre of four bonds with four chains each different from hydrogen, i.e. a point highly susceptible to scission under an ion beam. This ion is $\mathrm{Si}(\mathrm{OH})_{3}{ }^{+}$and an image of its signal has been constructed (Figure 7(a)). Although this image is of relatively low intensity, as the silane is a minor component of the system, one can see that the amount of $\mathrm{Si}(\mathrm{OH})_{3}{ }^{+}$is more intense within the adhesive than in the region where the aluminium is visible. A similar image was obtained for $\mathrm{AlOSi}^{+}$which is proof of bonding of the silane through condensation of a silanol with a hydroxyl functionality at the surface of the aluminium (Figure 7(b)). The image indicates that the intensity of $\mathrm{AlOSi}^{+}$seems more intense where the aluminium is most intense as this is the position where we are closest to the true interface and consequently the true location of such bonding reaction. The last reaction can be illustrated by the presence of an ion that would contain at least two silicon atoms and this is illustrated in Figure 7(c). This Figure shows that the region of crosslinking is partially overlapping with the region where $\mathrm{AlOSi}^{+}$is present but $\mathrm{Si}_{2} \mathrm{O}^{+}$is also more intense where PDMS peaks are found. This is because the same ion is characteristic of the silicone polymer (see Figure 5(c)).

In addition to the images already presented in this work, spectra were reconstructed from three regions of the image itself (Area 1) described as ROI 1, 2 and 3 (see Figure 5(c)), regions rich in PDMS (or pseudo PDMS peaks), one intermediate region with lower PDMS peak intensity and a region rich in aluminium respectively. The 
aluminium rich region has been determined on the basis of number of counts with a minimum of 125 counts after normalisation. Those spectra are not presented here because they are very similar to the other two spectra presented in Figure 4 but they indicate that ROI 2 exhibits some PDMS peaks of lower intensity than ROI 1 which seems mostly composed of silicone based material. When the spectrum of ROI 3 is examined, no PDMS like peaks are visible and its spectrum is similar to that obtained close to aluminium in Figure 4 with various ions assignable to $\mathrm{C}_{\mathrm{x}} \mathrm{H}_{\mathrm{y}}$ structures. The three silane reactions of interest may still be examined but the actual presence of PDMS in the most covered aluminium region casts a shadow on any data that may both originate from the silane's reactions or PDMS itself and will be examined in more detail in the discussion section. This is the case for $\mathrm{Si}_{2} \mathrm{O}^{+}$, in particular, the most simple fragment one may obtain for crosslinked APS.

\section{Discussion}

The XPS profiles indicate the diffusion of APS to the interface of the adhesive with aluminium foil, in particular when the profile of silicon is examined as it is the marker of the silane molecule. All other obvious sources of silicon(e) have been eliminated to achieve this. Such behaviour has already been shown in a very different system and for a different substrate: APS containing polyamide on steel $[3,4,5]$. In this work, APS is used too and migrates as well to the interface between the polyamide coating and the steel. Incidentally, APS migrates also to the air coating interface. This probably implies that should the model sample discussed in this work be used in a real adhesive joint, APS would migrate at both interfaces with the substrate of use. It is particularly interesting to notice that the diffusion seems to occur in a similar way, with similar silicon concentration (between 1 and 2 at.\%) and a similar front of diffusion of a few micrometers only. Considering that substrates are different (steel and aluminium) as well as the two media in which the silane has to migrate (a thermoplastic versus a thermoset) may also imply that the diffusion is characteristic of the molecule rather than the system examined or that the parameters controlling the diffusion are similar in both formulations. However, in both systems a diffusion probably occurs when either the thermoplastic coating or the adhesive is still in a soft/liquid form. The main difference resides in the temperature used for melting compared to cure temperature and also in the chemistry of the systems which do not 
seem to have a significant effect on the resulting diffusion. What remains to be explained are the driving forces leading to the segregation of silane at the interface. For such a phenomenon to occur, two conditions at least are required: the mobile molecules need to be able to move in the polymer matrix and the local enrichment has to be driven by a force. One argument that may be put forward is that the metal substrate acts as a "sink" for the silane molecules which are in random movement within the formulation as APS can interact with the substrate either with forming a covalent bond by condensation or by acid-base type of interaction which is possible at either end of the molecule $[6,7]$. The molecule can indeed form a bond as anticipated with condensation of a silanol and a hydroxyl functionality present at the surface of the aluminium substrate or interact through a hydrogen bond between again the hydroxyl and the unshared pair of electrons present on the nitrogen functionality of the same silane. Once the molecule interacts with the substrate further random movement, presumably augmented by heat, can bring more APS molecules in contact with the already interacting ones and eventually form a network of APS molecules either with a region of pure silane or diluted within the polymer adhesive matrix. Other factors to consider include the solubility parameters. If the parameters for the silane and polymer matrix are comparable, then the degree of interdiffusion is higher as shown in the work of Gentle et al [8]. The solubility parameter of APS is equal to

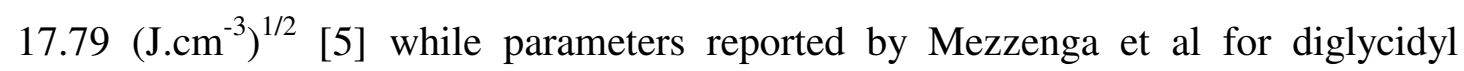
bisphenol A crosslinked stochiometrically with isophorone diamine vary between 17.06 and $17.92\left(\mathrm{~J} . \mathrm{cm}^{-3}\right)^{1 / 2}$ according to formulation and degree of conversion (percentage of reacted epoxy functionalities). It is important to note that the solubility parameter values increase with degree of conversion. Assuming that the behaviour of the resin used in this work is similar this would make the formulation and the APS silane more compatible with its polymer host as the resin crosslinks and therefore limit the diffusion as the adhesive hardens. The silane would be first pushed out of the mixture and then become compatible with time which could be one of the reasons why it segregates to the interface. This phenomenon is similar to that employed, for example, in such applications as self stratifying coatings for metallic substrates. Benjamin et al. [9] tried to use solubility parameters to explain self-stratification of a mixture of two polymers; they noted, however, that the use of solubility parameters was not sufficient to explain fully the occurrence (or absence) of segregation of one particular component. Nenakhov et al observed also that the diffusion of small 
molecules is only limited by the changes in molecular weight of the crosslinking resin if their volume fraction is below 0.5 [10]. Another possible explanation lies into the value of the chemical potential (partial molar Gibbs free energy) of APS within the adhesive, which is a thermodynamic quantity. For a system containing several components, there is a separate chemical potential associated with each species and matter flows spontaneously from a region of high chemical potential to a region of low chemical potential. In this case APS would have a high chemical potential within the adhesive and a lower one at interfaces, be it with the aluminium substrate or air, which would induce the diffusion.

The reaction of hydrolysis of APS (see Figure 6(a)) is illustrated and demonstrated by the presence of $\mathrm{Si}(\mathrm{OH})_{3}{ }^{+}$ion. An illustration of the presence of the ion $\mathrm{Si}(\mathrm{OH})_{3}{ }^{+}$is given in Figure 8. For the formation of this ion to occur, there must be presence of water within the system. It is quite likely that a small amount of water may be present within the formulation as well as on the aluminium foil itself and it is known that APS can also absorb atmospheric moisture. Aminosilanes are known as being selfcatalytic and therefore such an activity will facilitate reactions that are otherwise quite difficult. Water as a reactant is highly unlikely to be present in excess amount to allow displacement of the hydrolysis reaction towards "silanol" products. As mentioned above, the amount of $\mathrm{Si}(\mathrm{OH})_{3}{ }^{+}$is more intense where the adhesive is mostly present and then the intensity decreases when moving towards a stronger signal of aluminium. Similarly one can notice the reversed effect for $\mathrm{AlOSi}^{+}$ions. A possible explanation is that as silane bonding reactions occur on the aluminium; this ion is less visible as the chemical entity from which it is yielded is reduced. Such an entity may also be consumed through crosslinking reactions and may therefore also be an indication of the proximity of the silane molecules themselves.

Two types of covalent bonds formed by APS are considered in this work, the formation of a covalent bond between the aluminium substrate and the polymerisation of APS. There is also the possibility of the silane reacting with components of the adhesive itself but the ions generated in this way may have structures similar to that of the crosslinked adhesive and are therefore are rather difficult to isolate effectively. Besides the silicon profile indicates that most of the silane molecule migrates to the interface, the remaining silicon is present only within a few micrometres of the substrate surface. A very small amount of silicon, below 0.2 at $\%$, may be assigned to 
silane having reacted in the bulk. The bonding of the silane with the aluminium substrate is, on the other hand, easy to show. Figure 9 illustrates the ion at mass 71 for Areas 2 and 3. It is obvious that only Area 3 (close to aluminium) exhibits the $\mathrm{AlOSi}^{+}$ion. This statement is also true for ROI 2 and ROI 3. Although we are not showing the corresponding spectra here as the signals are rather noisy, the intensity of this ion is actually illustrated in the image of Figure 7(b). The $\mathrm{AlOSi}^{+}$ion has been shown in previous work to correspond to the bond formed between silane and substrate, but it has always raised the question of possible ion recombination [11]. Although the formation of this ion is not zero, it is highly unlikely within the selvedge region of the plasma induced by the primary ion impact. For example, in ROI 1, the region exhibiting a high amount of PDMS peaks, aluminium dominates the ion at mass 27 while a lot of silicon is readily available; yet no $\mathrm{AlOSi}^{+}$is obtained for this ROI indicating that the hypothesis of recombination is not valid. It is also of interest to see that the silane has not only hydrolysed but also reacted with the aluminium surface while being incorporated within a formulation. This, of course, also establishes that a covalent bond with aluminium may be obtained with APS as well as with GPS [11].

Following the reaction of bonding one should consider crosslinking/polymerisation of APS. As indicated from the spectra shown in this work (see Figure 4), PDMS or PDMS like peaks are present in various regions of the sandwich sample. Figure 10 shows superimposed spectra for nominal mass 73 for ROI 1, ROI 2 and ROI 3. This peak is usually a good indicator of PDMS presence as it may be assigned to the fragment generated by the end of chain $\mathrm{Si}\left(\mathrm{CH}_{3}\right)_{3}{ }^{+}$. In Table 1 are provided the corrected and normalised intensities of this ion for ROI 1, ROI 2 and ROI 3 as well as Areas 2 and 3. One can see that the intensity is decreasing markedly when ROI 1 is compared to ROI 2 and has all but gone in ROI 3 (14 counts actually correspond to noise level for the signal), the region immediately adjacent to pure aluminium. Although not shown here, similar remarks apply to other usually characteristic PDMS peaks present in the spectra obtained form those ROIs such as nominal mass 147, 207 and 281 while the intensity of silicon remains high and constant (the corrected normalised intensity of silicon is equal to 51525, 50343 and 45445 for ROIs 1, 2 and 3 respectively). One of the main difficulties therefore resides in assigning those peaks to contamination or to similar structures formed through the polymerisation of APS. 
In previous work, the current authors have shown that the presence of water (used for rinsing films) may induce the formation of a similar structure to PDMS when APS is polymerised whereas no such phenomenon is observed for an epoxy based adhesion promoter [12]. This behaviour seems to be specific to APS as even if water from the same container used for APS is used for GPS no PDMS signature is visible in a ToFSIMS spectrum. This could explain why PDMS like peaks are present even though great care was taken not to have contamination present on and within the samples. It is also anticipated that if PDMS is present as a contaminant, it covers the available surface to reduce the surface energy according to Gibbs free energy principle. However it seems rather strange that the only area where PDMS like peaks are not present is close to aluminium; considering that the surface energy of a metal is higher than a polymeric material the reverse would be more logical should the peaks be assigned to PDMS. To provide similar peaks to PDMS, APS should polymerise or exhibit pseudo crosslinks into a similar structure i.e. linear. This implies that, at least in part of the interface region APS does not polymerise in a network indicating that may be the concentration of APS is too low for this to happen (as in ROI 1). Besides, the equivalent concentration of silicon that would be obtained by XPS for Area 2 would be of less than 1 at.\% for silicon while the amount of carbon would be over 30 at.\% providing a ratio very far from the expected one to two for PDMS, thus indicating that the origin of silicon in this particular region may not be solely assigned to the presence of PDMS. The fragment ion $\mathrm{Si}_{2} \mathrm{O}^{+}(\mathrm{m} / \mathrm{z}=72$ nominal mass) as shown in Figure 11 for Areas 2 and 3, may be obtained from either PDMS or polymerised APS and while it is only indicative of the polymer for PDMS as such bonds are within its structure, for APS it is indicative of crosslinking between at least two hydrolysed APS molecules. As an assessment of the type of molecule being present in the images and spectra the ratio of the intensity of $\mathrm{Si}_{2} \mathrm{O}^{+}$to $\mathrm{Si}\left(\mathrm{CH}_{3}\right)_{3}{ }^{+}$(nominal mass 73 , usually the main observed PDMS peak) was calculated and compared to the same ratio obtained from the ION TOF database. The results are reported in Table 1. They seem to indicate that for the region rich in PDMS, either PDMS is truly present or the structure formed by polymerised PDMS is indeed very similar to that of this polymer. It is, however, also obvious from the values obtained that $\mathrm{Si}_{2} \mathrm{O}^{+}$is more intense than expected for the sole presence of PDMS for the interface region and the only other explanation is that it is present through crosslinking of APS obtained with self condensation. 


\section{Conclusions}

This work leads to a number of conclusions as follows:

- Diffusion of the silane towards the interface is proven and APS exhibits a very small interphase of only a few micrometres. As APS is not usually the adhesion promoter employed in this adhesive, this may explain why APS is not the silane of choice for this particular adhesive. The diffusion also seems related to the characteristics of APS rather than the substrate or the polymer matrix. It seems also that APS is present at the interface in a pure form rather than to form a true mixed phase which, again, might act against its use within the adhesive.

- Hydrolysis of APS occurs within the mixture and is advanced even though no water is added to the system (a small amount if probably present within the system). This is probably due to the fact that APS is "auto-catalysed" in its reactions because of the presence of an amine.

- Bonding of APS occurs at the interface as shown by the presence of $\mathrm{AlOSi}^{+}$ ion the intensity of which is anti-correlated to that of $\mathrm{Si}(\mathrm{OH})_{3}{ }^{+}$ion indicating that the chemical species yielding the latter ion is consumed to produce a bond yielding the former.

- It is also possible to show that APS crosslinks using the presence of the $\mathrm{SiO}_{2}{ }^{+}$ ion. Arguments are presented which also shed light on other possibilities leading to the formation of pseudo PDMS ions from APS, indicating that such ions should not be systematically attributed to PDMS contamination.

\section{Acknowledgements}

The authors acknowledge The Royal Society for provision of a University Research Fellowship to MLA, Huntsmann Advanced Materials for provision of adhesive and Mr Jerry Powell for his help and advice in the formulation of epoxy adhesives. 
Figures and Tables

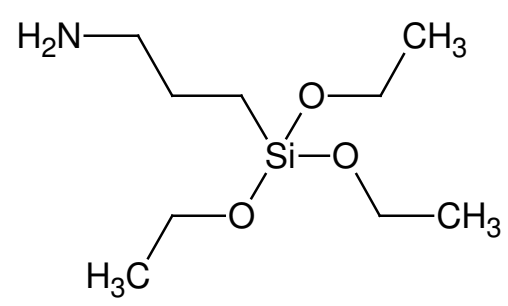

Figure 1: schematic of APS molecule

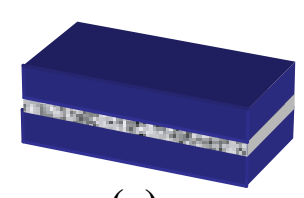

(a)

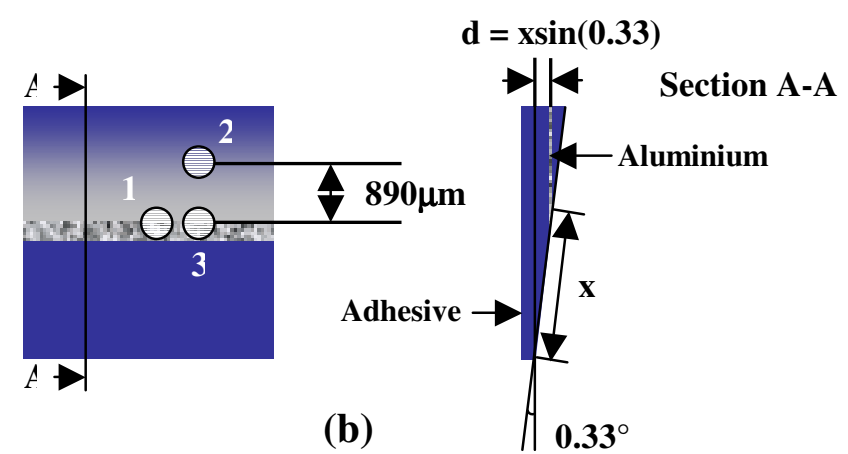

Figure 2: schematic of sandwich sample

(a) overall schematic; (b) plan and side elevation of the sectioned sample with analysis positions indicated. $x$ represents the distance of the XPS linescan while $d$ is the recalculated corresponding depth available to analysis through microtoming 


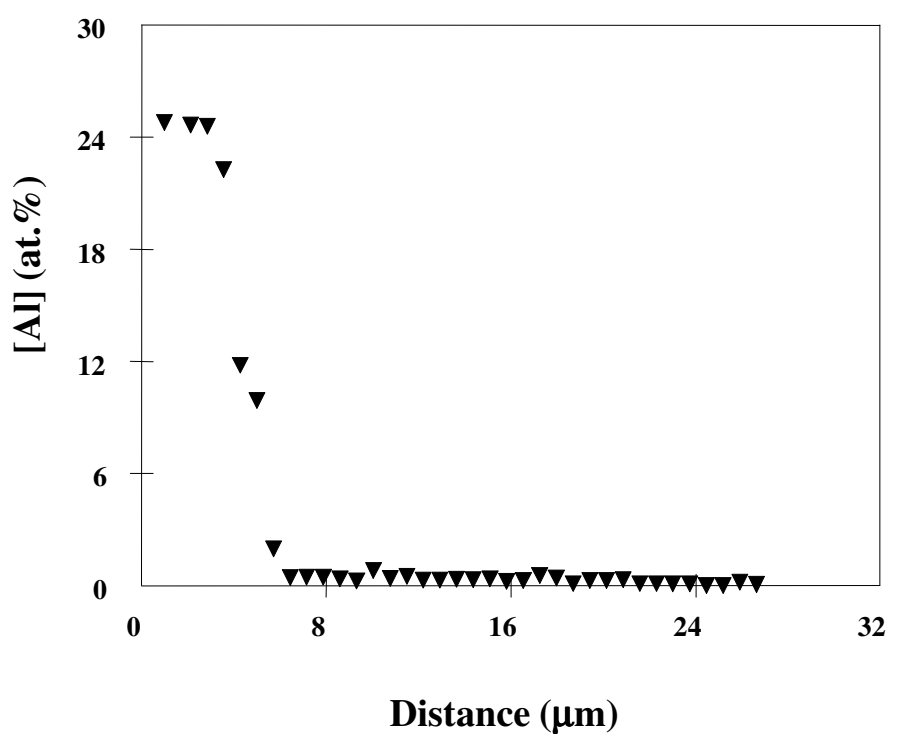

Figure 3(a): profile of aluminium concentration by XPS

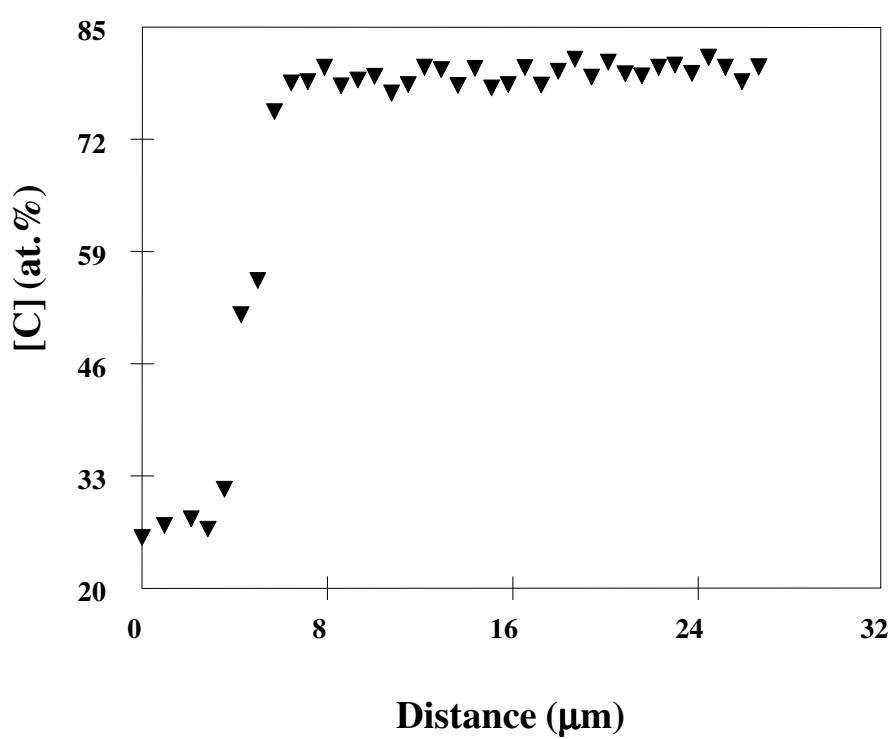

Figure 3(b): profile of carbon concentration by XPS 


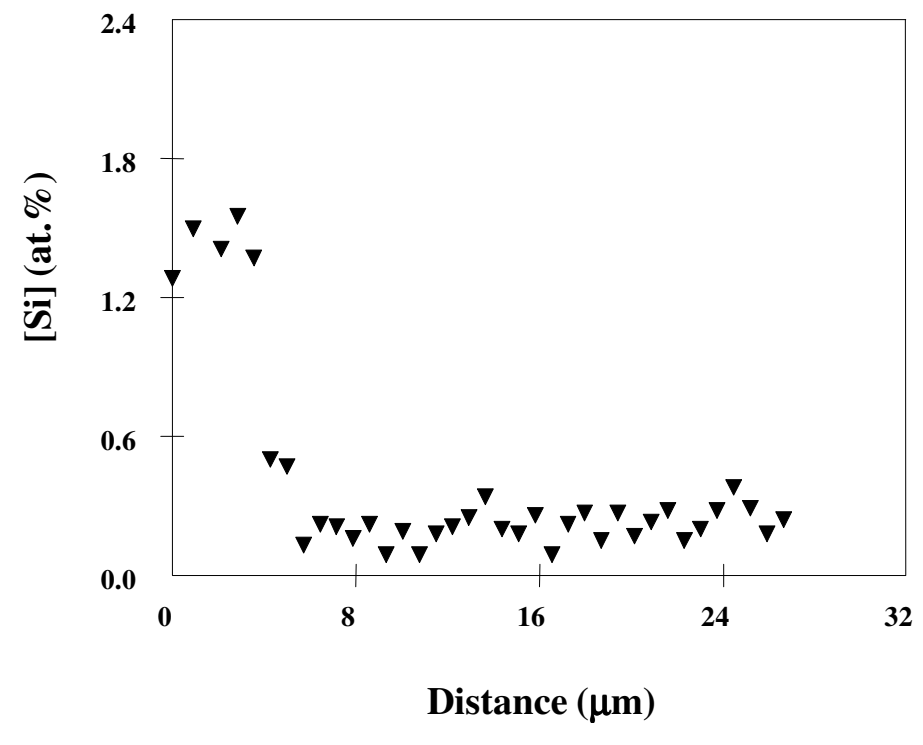

Figure 3(c): profile of silicon concentration by XPS

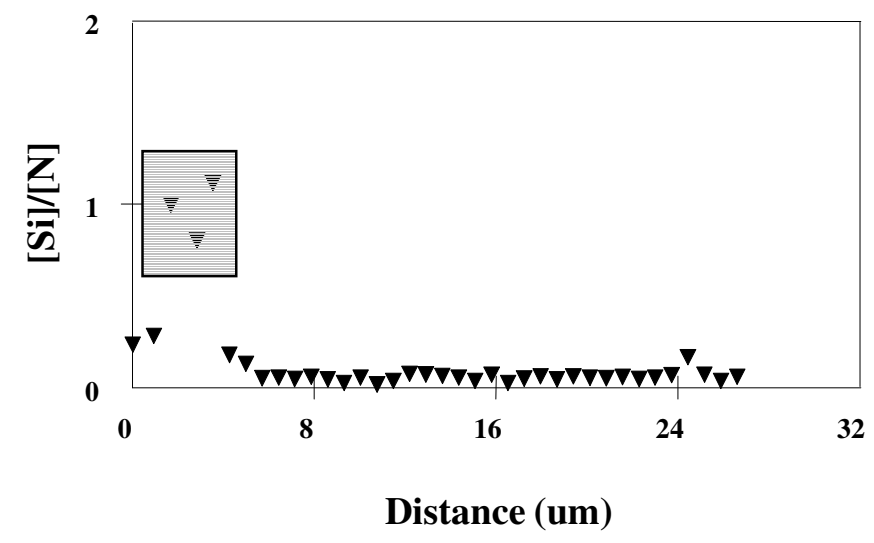

Figure 3(d): ratio of silicon to nitrogen concentrations 

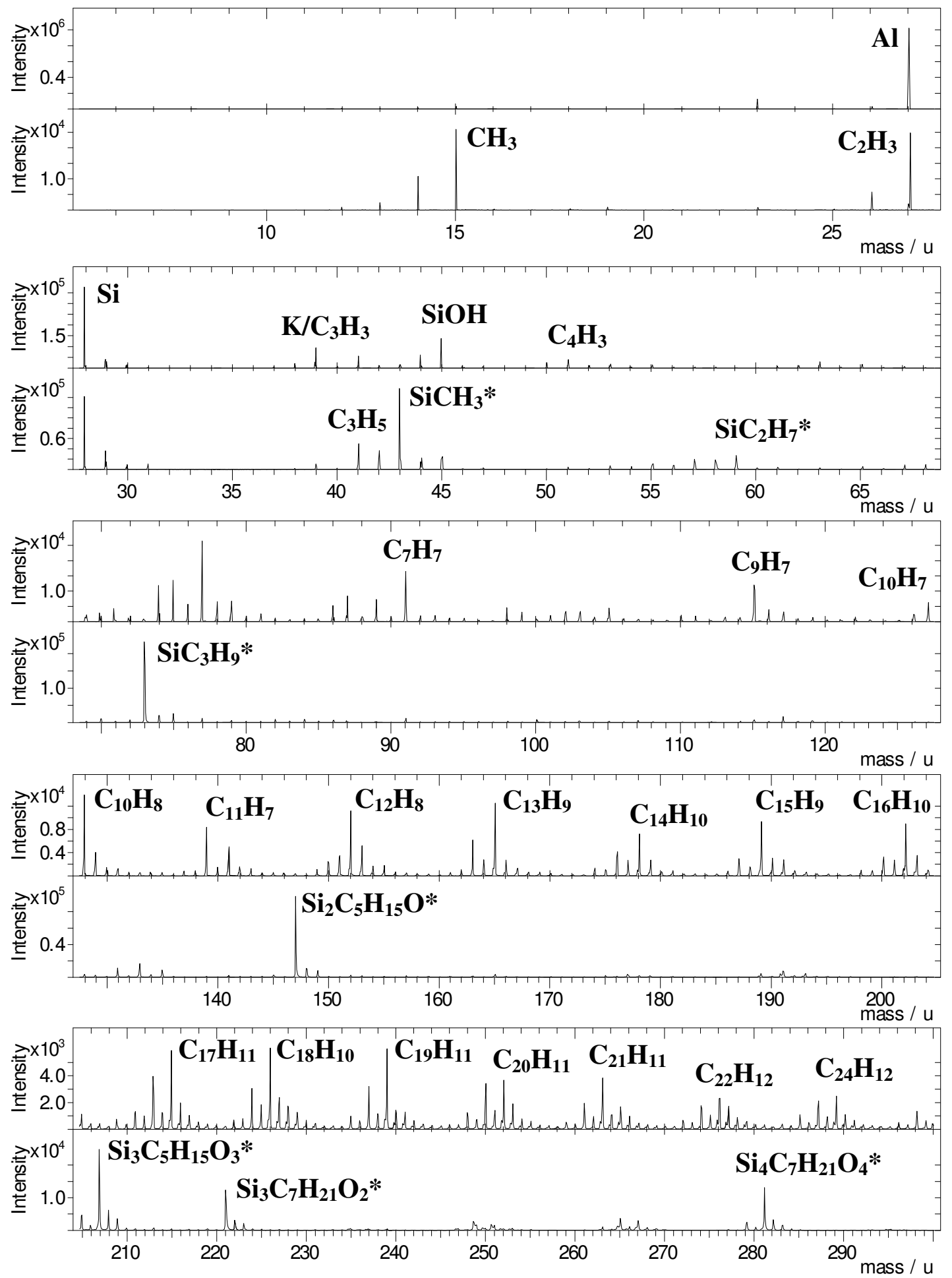

Figure 4: ToF-SIMS spectra obtained from area 3 (upper) and area 2 (lower) for masses (5-300u). PDMS like peaks are denoted by an asterisk*. 


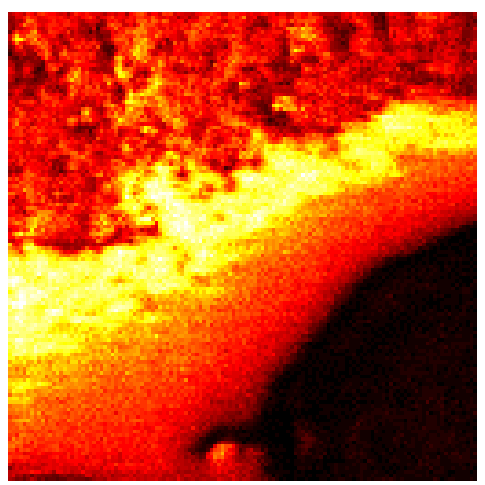

Figure 5(a): total ion signal obtained from the interface region

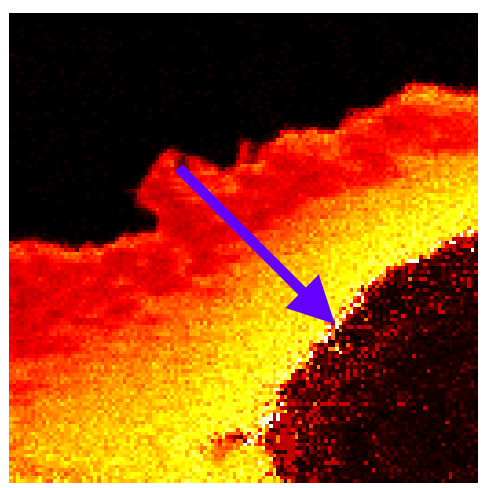

Figure 5(b): image of aluminium signal normalised to the total ion signal

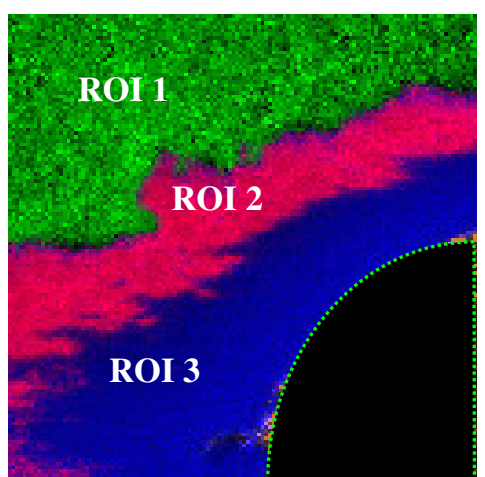

Figure 5(c): superimposition of PDMS rich signal (green) with low intensity PDMS signal (red) and aluminium signal (blue). The colour coding also corresponds to ROIs used for Table 1 and Figure 9. 


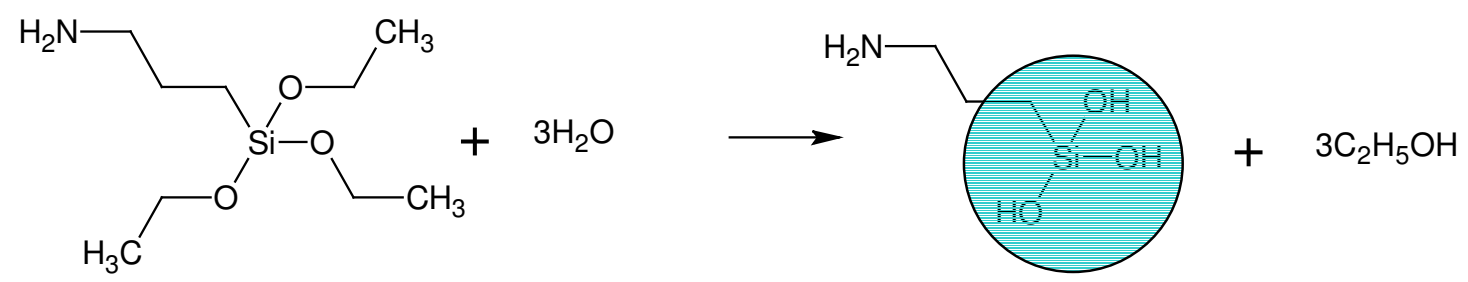

Figure 6(a): schematic of APS hydrolysis
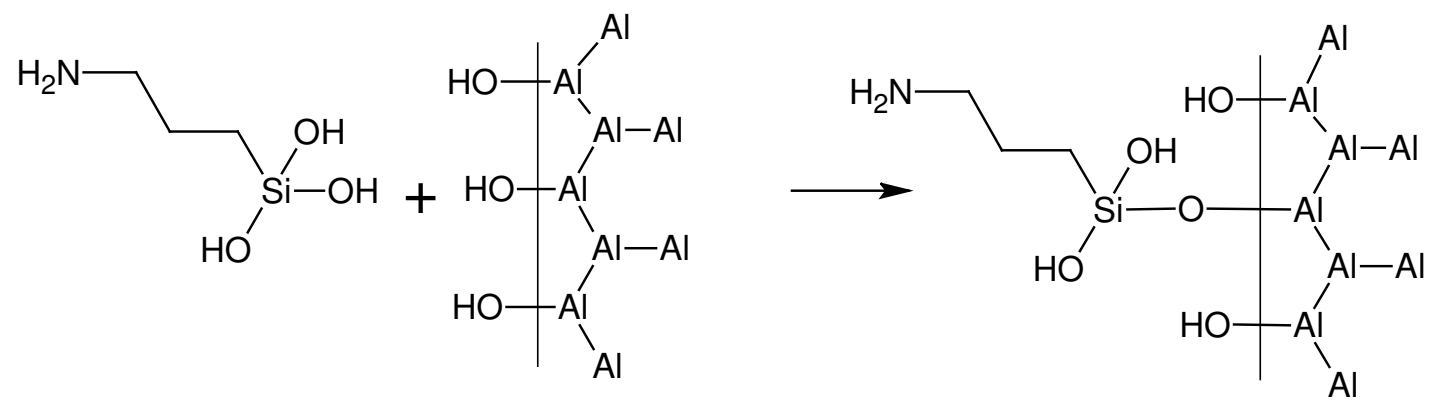

Figure 6(b): schematic of APS condensation with aluminium substrate

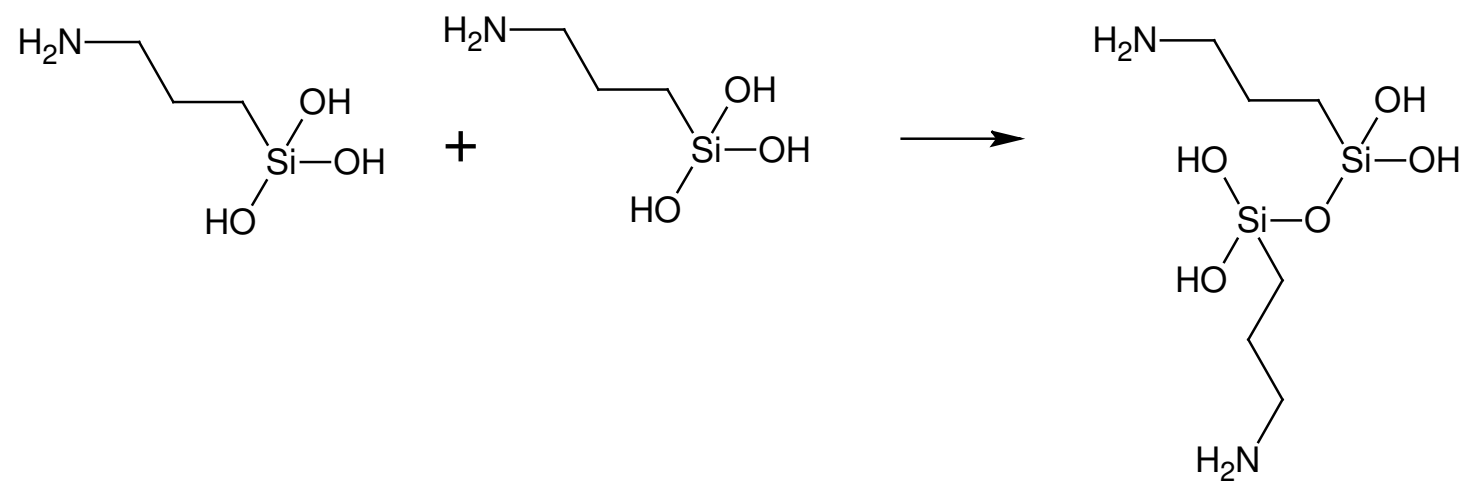

Figure 6(c): schematic of APS self-condensation (crosslinking) 


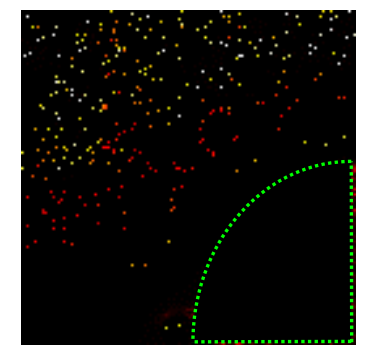

Figure 7(a): normalised image of $\mathrm{Si}(\mathrm{OH})_{3}{ }^{+}$

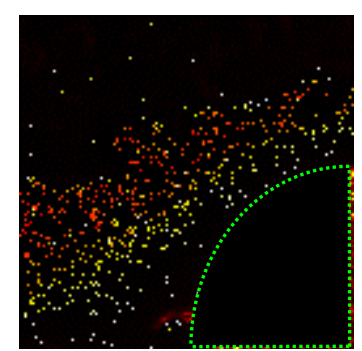

Figure 7(b): normalised image of $\mathrm{AlOSi}^{+}$

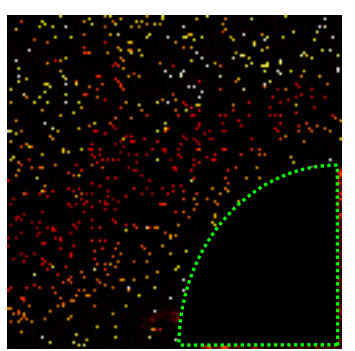

Figure 7(c): normalised images of $\mathrm{Si}_{2} \mathrm{O}^{+}$ 


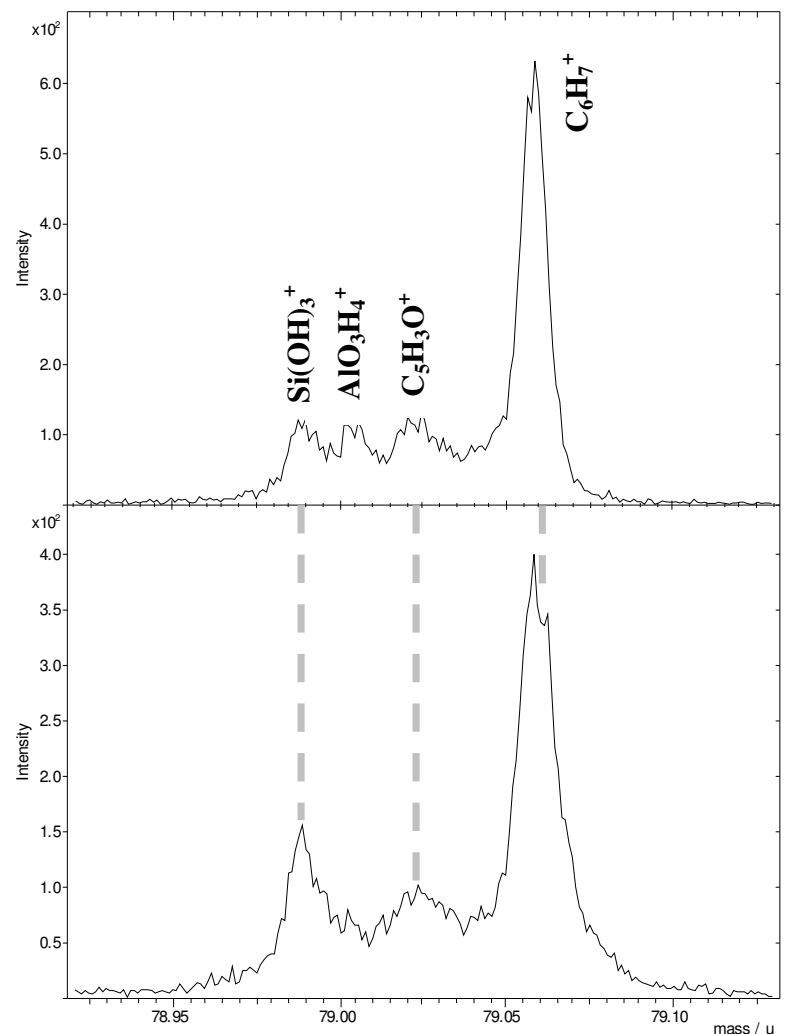

Figure 8: comparison of the peaks at nominal mass 79:

(a) Area 3 (b) Area 2 (see Figure 2) 


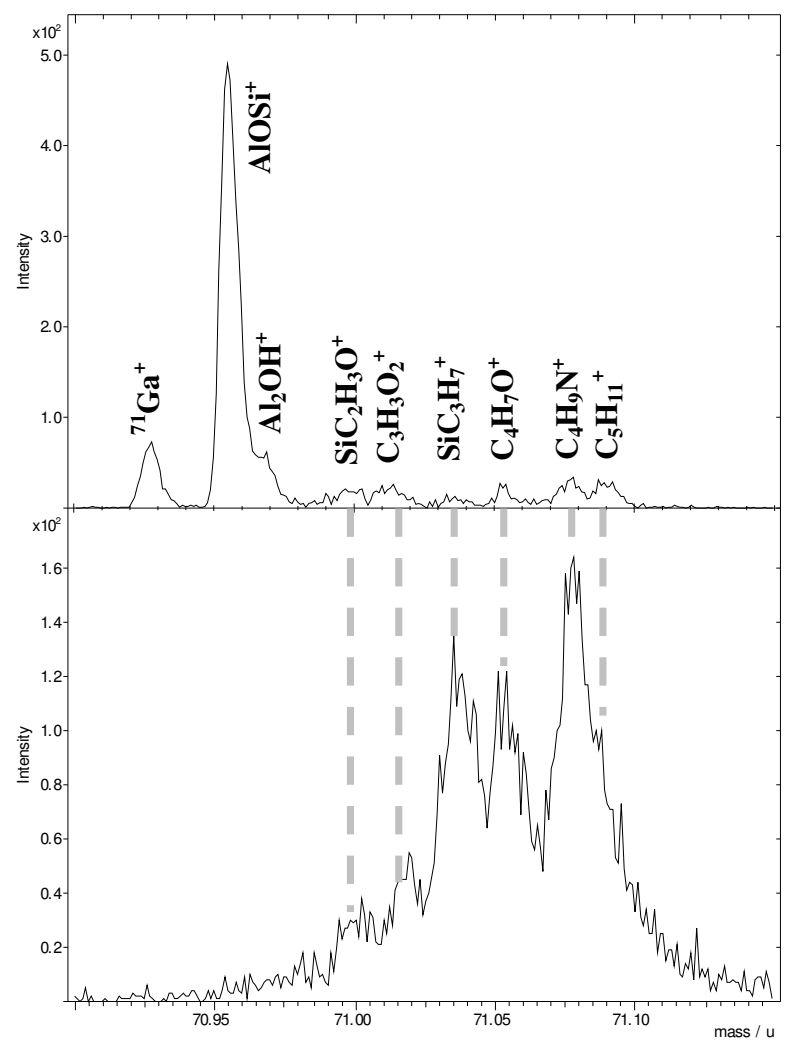

Figure 9: comparison of the peaks at nominal mass 71:

(a) Area 3 (b) Area 2 (see Figure 2) 


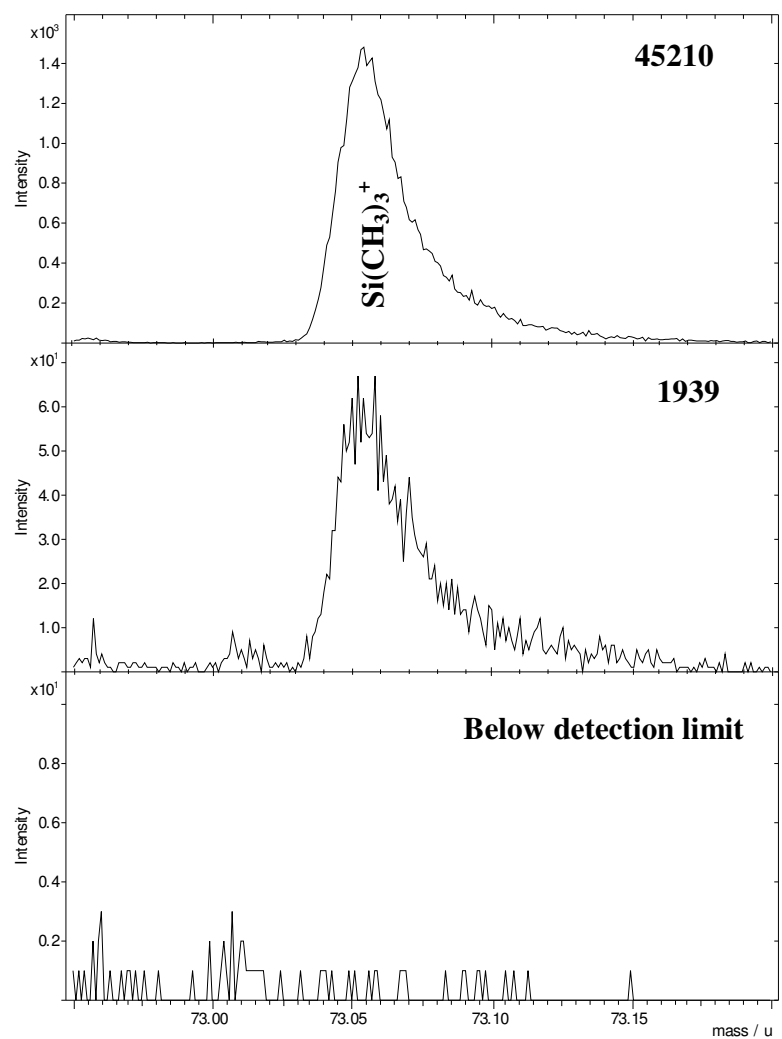

Figure 10: a comparison of peak 73 intensity for ROI 1, ROI 2 and ROI 3. 


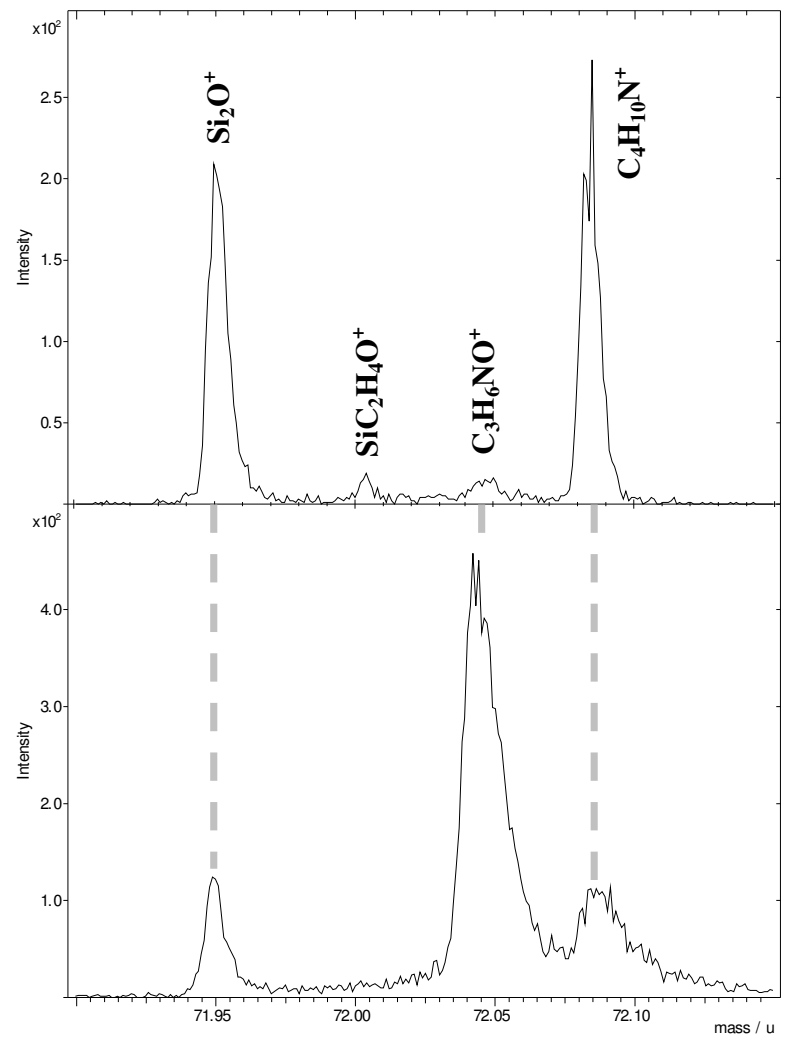

Figure 11: Comparison of the peaks at nominal mass 72: (a) Area 3 (b) Area 2 
Table 1: intensity ratios of $\mathrm{Si}_{2} \mathrm{O}^{+}$to $\mathrm{Si}\left(\mathrm{CH}_{3}\right)_{3}{ }^{+}$

\begin{tabular}{|c|c|c|c|}
\hline Region of sample & $\mathbf{S i}_{2} \mathbf{O}^{+}$ & $\mathbf{S i}\left(\mathbf{C H}_{3}\right)_{3}{ }^{+}$ & Ratio \\
\hline Interface with coating (spectrum Area 2) & 1339 & 393784 & 0.003 \\
\hline Region rich in PDMS (image ROI 1) & $\mathbf{2 8 1}$ & $\mathbf{4 3 3 6 5}$ & $\mathbf{0 . 0 0 6}$ \\
\hline $\begin{array}{c}\text { Intermediate region moderate amount of } \\
\text { PDMS (image ROI 2) }\end{array}$ & 230 & 1705 & 0.13 \\
\hline Region rich in aluminium (image ROI 3) & 153 & 14 & 10.9 \\
\hline Al interface area (spectrum Area 3) & 1905 & 634 & 3.0 \\
\hline Pure PDMS from ION TOF database & $\mathbf{0 . 3 6 - 0 . 4 9 *}$ & $\mathbf{8 0 . 9 3 - 1 0 0 *}$ & $\mathbf{0 . 0 0 4 4 - 0 . 0 0 4 9}$ \\
\hline
\end{tabular}

*Proportion of ion yield in percentages normalised to the highest intensity ion, respectively $\mathrm{Si}^{+}$ and $\mathrm{Si}\left(\mathrm{CH}_{3}\right)_{3}{ }^{+}$from PDMS as provided by the ION TOF database.

\section{References}

1. S. J. Hinder, C. Lowe, J. T. Maxted, J. F. Watts Surf. Interf. Anal. 12 (2004) 15751581 .

2. S. J. Hinder, C. Lowe, J. T. Maxted, J. F. Watts J. Mat. Sc. 40 (2005) 285-293.

3. M. Guichenuy, J. F. Watts, M-L. Abel, A. M. Brown, M. Audenaert, N. Amouroux, Surf. Interf. Anal., 36 (2004) 765-768

4. M. Guichenuy, J. F. Watts, M-L. Abel and M. Audenaert, Surf. Interf. Anal. 38 (2006) 168-171

5. Marianne Guichenuy, PhD Thesis dissertation, University of Surrey 2006

6. M. R. Horner, F. J. Boerio and H. M. Clearlfield in Silanes and Other Coupling Agents, pp 241-262, Ed. K. L. Mittal, VSP 1992

7. I. George, P. Viel, C. Bureau, J. Suski and G. Lecayon, Surf. Interf. Anal. 24 (1996) 774-780

8. T. M. Gentle, R. G. Schmidt, B. M. Naasz, A. J. Gellman, J. Adhes. Sci. Technol. 6 (1992) 307-316

9. S. Benjamin, C. Carr and D. J. Walbridge, Prog. Org. Coat. 28 (1996), pp 197-207

10. S. A. Nenakhov, A. Ye. Chalikh and A. T. Sanzharovskii, Polym. Sc. USSR 17 (1975) 1489-1497.

11. M-L. Abel, R. P. Digby, I. W. Fletcher and J. F. Watts, Surf. Interf. Anal. 29 (2000) 115-125.

12. M-L. Abel, J.F. Watts and R.P. Digby, Int. J. Adhes. Adhes. 18, (1998) 179-192.

\footnotetext{
${ }^{1}$ THE MÜNSTER HIGH MASS RESOLUTION STATIC SIMS LIBRARY. Print Edition V1.3 Volume II. Polymers. Copyright (C 1997-1999 by ION-TOF GmbH.
} 